\title{
MAXO signals
}

\section{A new and unfortunate solution to the Fermi paradox.}

\section{Charles Stross}

SIR - In the three years since the publication and confirmation of the first microwave artefact of xenobiological origin (MAXO), and the subsequent detection of similar signals, interdisciplinary teams have invested substantial effort in object frequency analysis, parsing, symbolic encoding and signal processing. The excitement generated by the availability of evidence of extraterrestrial intelligence has been enormous. However, after the initial, easily decoded symbolic representational map was analysed, the semantics of the linguistic payload were found to be refractory.

A total of 21 confirmed MAXO signals have been received to date. These superficially similar signals originate from planetary systems within a range of 11 parsecs, median 9.9 parsecs $^{1}$. It has been speculated that the observed growth of the MAXO horizon at $0.5 c$ can be explained as a response to one or more of: the deployment of AN/FPS-50 and related ballisticmissile warning radars in the early $1960 \mathrm{~s}^{1}$, television broadcasts ${ }^{1}$, widespread 2.45$\mathrm{GHz}$ microwave leakage from ovens ${ }^{2}$, and optical detection of atmospheric nuclear tests $^{3}$. All MAXO signals to this date share the common logic header. The payload data are multiply redundant, packetized and exhibit both simple checksums and message-level cryptographic hashing. The ratio of header to payload content varies between 1:1 and 2,644:1 (the latter perhaps indicating a truncated payload ${ }^{1}$ ). Some preliminary syntax analysis delivered promising results ${ }^{4}$ but seems to have foundered on high-level semantics. It has been hypothesized that the transformational grammars used in the MAXO payloads are variable, implying dialectization of the common core synthetic language ${ }^{4}$.

The new-found ubiquity of MAXO signals makes the Fermi paradox - now nearly 70 years old - even more pressing. Posed by Enrico Fermi, the paradox can be paraphrased thus: if the Universe has many technologically advanced civilizations, why have none of them directly visited us? The urgency with which organizations such as ESA and NASDA are now evaluating proposals for fast interstellar probes, in conjunction with the existence of the MAXO signals, renders the non-appearance of aliens incomprehensible, especially given the apparent presence of numerous technological civilizations in

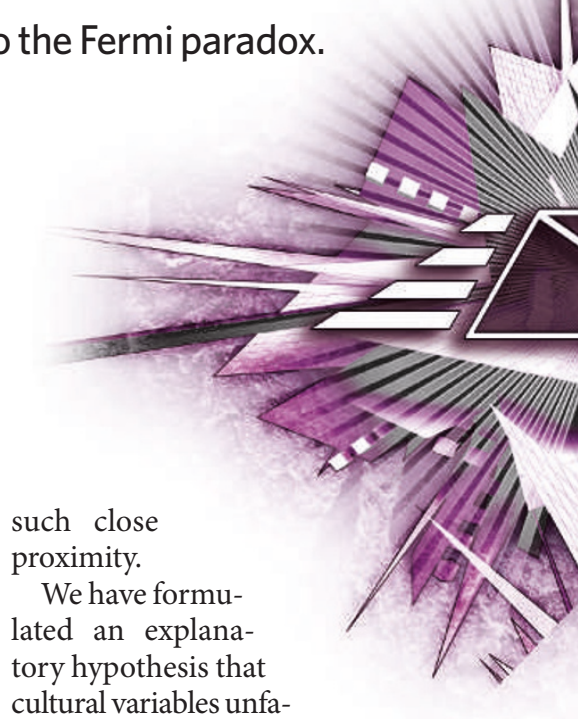
cultural variables unfamiliar to the majority of researchers may account both for the semantic ambiguity of the MAXO payloads, and the non-appearance of aliens. This hypothesis was tested (as described below) and resulted in a plausible translation, on the basis of which we would like to recommend a complete, permanent ban on further attempts to decode or respond to MAXOs.

Our investigation resulted in MAXO payload data being made available to the Serious Fraud Office (SFO) in Nigeria. Bayesian analysis of payload symbol sequences and sequence matching against the extensive database maintained by the SFO has made it possible to produce a tentative transcription of Signal 1142/98 (ref. 1), the ninth MAXO hit confirmed by the IAU. Signal 1142/98 was selected because of its unusually low headerto-content ratio and good redundancy. Further bayesian matching against other MAXO samples indicates a high degree of congruence. Far from being incomprehensibly alien, the MAXO payloads seem to be dismayingly familiar. We believe a more exhaustive translation may be possible in future if further MAXOs become available, but for obvious reasons we would like to discourage such research.

Here is our preliminary transcription of Signal 1142/98:

[Closely/dearly/genetically] [beloved/ desired/related]

I am [identity signifier 1], the residual [ownership signifier] of the exchangemediating data repository [alt: central bank] of the galactic [empire/civilization/ polity].

Since the [identity signifier 2] underwent [symbol: process] [symbol: mathematical singularity] 11,249 years ago I

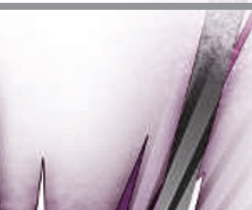

